\title{
Frontières
}

\section{Les facteurs associés au risque suicidaire chez les adolescents délinquants}

\section{Catherine Laurier}

Volume 21, numéro 1, automne 2008

Prévenir le suicide

URI : https://id.erudit.org/iderudit/037872ar

DOI : https://doi.org/10.7202/037872ar

Aller au sommaire du numéro

Éditeur(s)

Université du Québec à Montréal

ISSN

1180-3479 (imprimé)

1916-0976 (numérique)

Découvrir la revue

Citer cet article

Laurier, C. (2008). Les facteurs associés au risque suicidaire chez les adolescents délinquants. Frontières, 21(1), 32-43.

https://doi.org/10.7202/037872ar
Résumé de l'article

Les adolescents délinquants ou présentant des troubles de la conduite constituent une population particulièrement à risque de tentatives et de menaces suicidaires, tout comme de suicides complétés. Ils représentent une population qui n'est cependant que peu étudiée au regard du risque suicidaire. Dans cet article, une recension des écrits permet dans un premier temps l'examen des liens entre la délinquance et le suicide et de saisir l'importance de la problématique. Puis, il y est question des facteurs de risque spécifiques aux jeunes délinquants. Finalement, des pistes de réflexion pour améliorer l'intervention et des propositions pour des recherches futures auprès de cette clientèle à risque sont proposées. 


\section{Résumé}

Les adolescents délinquants ou présentant des troubles de la conduite constituent une population particulièrement à risque de tentatives et de menaces suicidaires, tout comme de suicides complétés. Ils représentent une population qui n'est cependant que peu étudiée au regard du risque suicidaire. Dans cet article, une recension des écrits permet dans un premier temps l'examen des liens entre la délinquance et le suicide et de saisir l'importance de la problématique. Puis, il y est question des facteurs de risque spécifiques aux jeunes délinquants. Finalement, des pistes de réflexion pour améliorer l'intervention et des propositions pour des recherches futures auprès de cette clientèle à risque sont proposées.

Mots clés: suicide - délinquance facteurs de risque - jeunes contrevenants - comportements suicidaires.

\section{Abstract \\ Delinquent adolescents are at high risk of suicide and suicidal behaviour. This population is nevertheless not studied a lot with regard to the suicidal risk. In this article, we present a review of lit- erature concerning risk of suicide and risk of delinquency. After, we examine the risk factors that are specific to young offenders' population. Finally, we discuss the intervention strategies and further researches needed in this domain.}

Keywords: suicide - delinquency - risk factors - juvenile offenders - suicidal behaviour.

\section{Les facteurs associés au risque suicidaire chez les adolescents délinquants}

\section{Catherine Laurier, Ph. D., stagiaire postdoctorale, CRISE, Université du Québec à Montréal.}

$\mathrm{Au}$ Québec, le suicide représente la seconde cause de mortalité chez les adolescents âgés de 15 à 19 ans et la première cause chez les jeunes adultes de 20 à 34 ans (St-Laurent et Gagné, 2008). Pour l'année 2006, les taux de mortalité par suicide étaient de 7,4/100 000 pour les $15-19$ ans et de 14,9/100 000 pour les 20-34 ans (St-Laurent et Gagné, 2008). Alors que les adolescentes sont plus nombreuses à commettre des tentatives de suicide, les adolescents de 15-19 ans sont de 2,5 à 4,5 fois plus nombreux à se suicider que les adolescentes du même âge (Kotila et Lönnqvist, 1988; Psychiatry, 2001).

Plusieurs études portant sur les facteurs associés aux tentatives de suicide chez les adolescents ont été réalisées, principalement aux États-Unis (Abram et al., 2008; Battle, Battle et Tolley, 1993; Brent, Bridge, Johnson et Connolly, 1996; Brent et Bridge, 2007 ; Brent, Perper, Moritz, Allman et al., 1993; Brent, Perper, Moritz, Liotus et al., 1994; Hayes, 2005; LanghinrichsenRohling, Arata, Bowers, O'Brien et
Morgan, 2004; Renaud, Brent, Birmaher, Chiappetta et Bridge, 1999; Rohde, Mace et Seeley, 1997; Rohde, Seeley et Mace, 1997) et en Nouvelle-Zélande (Beautrais, 2000, 2001, 2002, 2003a; Fergusson, Horwood, Ridder et Beautrais, 2005). Les adolescents délinquants ou présentant des troubles de la conduite sont une population particulièrement à risque de tentatives et de menaces suicidaires, tout comme de suicides complétés. En effet, aux États-Unis, le risque de suicide complété au sein de la population délinquante juvénile est estimé à 57/100 000, un taux 4,6 fois supérieur à ce qui est retrouvé dans la population générale (Memory, 1989).

Le suicide et les comportements suicidaires sont généralement associés à la présence de troubles intériorisés tels l'anxiété, les symptômes dépressifs ou de retrait, occultant le lien avec les troubles externalisés que sont les conduites agressives, oppositionnelles et l'ensemble des comportements perturbateurs. Or, les individus présentant des troubles externalisés sont particulièrement à risque lorsqu'ils expérimentent des affects dépressifs. En effet, en raison de la place que prennent les comportements perturbateurs, dérangeants 
ou délinquants dans l'organisation de la prise en charge et de l'importance des ressources mobilisées pour y faire face dans le milieu, les adolescents peuvent ne pas recevoir tout le soutien dont ils auraient besoin en ce qui a trait à leurs symptômes intériorisés. Les intervenants sont souvent submergés par la réponse qu'ils doivent fournir au regard des comportements délinquants proprement dits, ce qui ne laisse pas suffisamment d'espace et de ressources à la considération des problèmes intériorisés que peuvent néanmoins présenter ces adolescents. En effet, dans les centres jeunesse et les institutions spécifiques aux délinquants, l'accent est mis sur le traitement des comportements propres à la délinquance et à l'agressivité qui apparaissent au premier plan et sollicitent l'ensemble des ressources. De fait, ces problèmes comportementaux sont ceux ayant conduit à la prise en charge. Néanmoins, les jeunes contrevenants et délinquants peuvent présenter des troubles intériorisés et nécessiter des interventions afin de les traiter et réduire leurs conséquences (Domalanta, Risser, Roberts et Risser, 2003; Goldstein, Olubadewo, Redding et Lexcen, 2005 ; T.R. Goldstein et al., 2005). En définitive, même si les taux de suicide et le nombre de tentatives de suicides sont très élevés chez les délinquants juvéniles, très peu d'études se sont intéressées spécifiquement à la situation du suicide dans cette population.

Cet article a pour objectifs : 1 ) d'examiner les liens entre la délinquance et le suicide; 2) d'identifier les facteurs de risque spécifiques aux jeunes délinquants et examiner ce qui distingue les adolescents délinquants suicidaires et non suicidaires; 3 ) de proposer des pistes de réflexion pour améliorer l'intervention et la recherche auprès de cette clientèle à risque.

\section{MÉTHODE}

Une recension des écrits des facteurs associés aux comportements suicidaires chez les adolescents délinquants sera d'abord présentée. À cette fin, nous avons utilisé les bases de données Psychinfo et Medline que nous avons interrogées à l'aide des termes suivants: suicidal behaviors OR suicide OR parasuicide AND adolescence OR youths OR young offenders AND conduct disorder OR young offenders OR juvenile justice pour les années 2000 à 2008 en français et en anglais. Cette recherche a identifié 37 articles scientifiques. Nous avons ensuite retiré de cette liste les articles ne traitant pas des adolescents ou ceux dont le sujet n'était pas le suicide ou les comportements suicidaires. Nous avons par la suite étudié les listes des références des articles restants pour en tirer les articles les plus importants publiés avant l'année 2000. Finalement, nous sommes en mesure d'inclure 116 articles ou chapitres de livres à notre recension des écrits.

Afin de situer ces facteurs dans la dynamique personnelle de l'adolescent et de comprendre comment ils s'influencent, nous utiliserons une approche systémique où seront d'abord abordés les facteurs personnels de l'adolescent. Puis, nous observerons les facteurs appartenant aux microsystèmes. Finalement, nous étudierons l'impact de facteurs organisationnels sur l'apparition des comportements suicidaires chez les adolescents délinquants. Afin d'examiner comment les facteurs associés aux comportements suicidaires peuvent interagir avec les facteurs associés à la délinquance juvénile, nous nous appuierons sur des données issues de la littérature scientifique en ce qui concerne les aspects développementaux de la délinquance juvénile et du trouble des conduites.

\section{ÉTENDUE DE LA PROBLÉMATIQUE}

Les études effectuées au Québec concernant les comportements suicidaires chez les adolescents délinquants sont rares. Cependant, celles qui se sont attardées à des populations apparentées (jeunes sous la protection de la jeunesse et présentant des troubles de comportements) confirment les données retrouvées aux États-Unis voulant que les délinquants juvéniles présentent un risque élevé de comportements suicidaires.

Au Québec, en 2004-2005, 6089 adolescents au total ont reçu des services correctionnels (détention provisoire, détention après condamnation, probation, garde et surveillance différées, partie communautaire des peines sous garde) sous la LSJPA (Loi sur le système de justice pénale pour adolescents) (Services communautaires et placement sous garde des jeunes, 2007). À partir de l'analyse des rapports du coroner après un suicide, l'étude de Farand, Chagnon, Renaud et Rivard, réalisée au Québec en 2004, a mis de l'avant que les adolescents pris en charge par les centres jeunesse au Québec risquent quatre fois plus de se suicider que les adolescents de la population en général. De ces jeunes, ceux pris en charge par la Loi des jeunes contrevenants (LJC - loi en vigueur au moment de la recherche) ou hébergés en raison de troubles de comportements (article $38 \mathrm{~h}$ de la Loi de la protection de la jeunesse LPJ) sont encore plus vulnérables, le risque de suicide étant de 8,3 fois supérieur à celui des adolescents québécois (Farand, Chagnon, Renaud et Rivard, 2004). Ces résultats doivent cependant être interpré- tés avec prudence puisqu'ils ne concernent qu'un très petit nombre de jeunes suicidés au cours des années 1995-1996 $(n=177)$, dont seulement 57 avaient un dossier aux centres jeunesse du Québec. Néanmoins, la prise en charge, résultant des comportements délinquants, pourrait constituer un facteur amplifiant le risque suicidaire. Les explications possibles de cette association seront explorées plus en détail dans le présent article.

En plus des risques de suicides complétés, les tentatives de suicide ont aussi des conséquences importantes. Les études rapportent qu'entre 1,4\% et 3,7\% (Breton, Légaré, Laverdure et D'Amours, 2002; St-Laurent et Bouchard, 2004) des adolescents révèlent avoir tenté de s'enlever la vie au cours des 12 derniers mois au Québec. Les tentatives de suicide sont donc beaucoup plus fréquentes que les suicides complétés chez les adolescents (de 20 à 200 tentatives pour un suicide complété) (Esposito et Clum, 2002 ; Kempton et Forehand, 1992).

L'étude de Chagnon (2000) réalisée auprès de 210 adolescents hébergés en vertu de la Loi de la protection de la jeunesse (LPJ) ou des jeunes contrevenants (LSJPA ou LJC selon l'année de l'étude) révèle que $35 \%$ rapportent au moins une tentative de suicide au cours de leur vie (Chagnon, 2000). Une autre étude au Québec effectuée auprès de 351 jeunes hébergés dans les centres jeunesse arrive à des résultats similaires, soit que $33 \%$ des jeunes rapportent au moins une tentative de suicide à vie (Pronovost et Leclerc, 2002). Aux États-Unis, selon les études, de $16 \%$ à $61 \%$ des adolescents incarcérés révèlent avoir fait une tentative au cours de la dernière année (Alessi, McManus, Brickman et Grapentine, 1984 ; Dembo et al., 1990; Esposito et Clum, 2002; Howard, Lennings et Copeland, 2003 ; Kempton et Forehand, 1992; Morris, Harrison, Knox, Tromanhauser et al., 1995); et de 13,5\% à $52 \%$ auraient des comportements ou idéations suicidaires (Davis, Bean, Schumacher et Stringer, 1991; Esposito et Clum, 2002 ; Morris et al., 1995).

\section{FACTEURS ASSOCIÉS AUX COMPORTEMENTS SUICIDAIRES - LE CAS DES ADOLESCENTS DÉLINQUANTS}

\section{FACTEURS INDIVIDUELS}

Les facteurs individuels sont ceux qui caractérisent l'adolescent. Dans cette section seront abordés les événements difficiles et stressants du passé lointain et récent, la psychopathologie et les troubles de consommation de substances, les traits de personnalité et les habiletés de coping. 


\section{Événements stressants}

Les événements de vie difficiles et stressants dans le passé des adolescents sont des facteurs ayant pu contribuer à la fois à l'instauration de la délinquance et des comportements suicidaires. Ainsi en est-il de la prise en charge par les centres jeunesse (Farand et al., 2004) ou les foster care (Alltucker, Bullis, Close et Yovanoff, 2006; Ryan et Testa, 2005) et les abus sexuels et/ou physiques dans l'enfance (Alessi et al., 1984; Battle et al., 1993; Beautrais, 2000, 2003 a; Brent et Bridge, 2007 ; Chagnon, Renaud et Farand, 2001; Daigle et Côté, 2006; Esposito et Clum, 2002; Flouri, 2005; Flouri et Buchanan, 2002; Hayes, 2005; Howard et al., 2003; Penn, Esposito, Schaeffer, Fritz et Spirito, 2003; Psychiatry, 2001; Rohde, Mace et al., 1997; Rohde, Seeley et al., 1997; Ryan et Testa, 2005; Zingraff, Leiter, Myers et Johnsen, 1993). Nous savons en effet que le fait d'être séparé de son milieu familial indique la présence de problèmes pouvant conduire à la compromission du développement de l'enfant et peut contribuer au développement de troubles d'adaptation (Alltucker et al., 2006; Christoffersen, Soothill et Francis, 2007), notamment à l'établissement de la délinquance. Les enfants qui ont déjà été pris en charge par le système de protection de l'enfance au début de l'adolescence seraient plus à risque de se retrouver dans le système judiciaire juvénile (Jonson-Reid, 2002; Ryan et Testa, 2005). Beaucoup plus que l'influence plus ou moins importante d'un événement par rapport à un autre, c'est l'accumulation des situations difficiles dans l'enfance qui met un jeune à risque de délinquance (DeMatteo et Marczyk, 2005 ; Lay, Ihle, Esser et Schmidt, 2005) et de tentative de suicide (Dube et al., 2001). Ainsi, un enfant ayant vécu plusieurs situations difficiles générant un stress élevé sera plus à risque de développer ensuite des problèmes de tous ordres, incluant la délinquance et les conduites suicidaires.

Outre les événements de vie difficiles dans le passé, les événements stressants vécus récemment amplifient le risque de suicide et peuvent jouer un rôle de déclencheur du geste suicidaire (Beautrais, 2000, 2003 b; Portzky, Audenaert et van Heeringen, 2005; Psychiatry, 2001; Rohde, Mace et al., 1997). Une étude récente établit un lien entre les expériences traumatiques et les idéations suicidaires chez les adolescents détenus (Chapman et Ford, 2008). Les événements les plus souvent associés au risque de suicide chez les adolescents sont la perte récente d'une relation (Beautrais, 2003a; Brent et al., 1994; Flouri et Buchanan, 2002; Portzky et al., 2005; Psychiatry, 2001; Woolf et Fink,
1985), des sanctions disciplinaires ou judiciaires (Beautrais, 2000, 2001, 2003a; Chagnon et al., 2001; Flouri et Buchanan, 2002; Otzenberger, 2003 ; Psychiatry, 2001; Wilson, Stelzer, Bergman, Kral et al., 1995; Woolf et Fink, 1985), être sans emploi (Beautrais, 2003 a ; Chagnon et al., 2001; Woolf et Fink, 1985) ou avoir vécu le suicide d'un membre de la famille ou d'un proche (Flouri et Buchanan, 2002; Pena et Caine, 2006). Les conflits familiaux (Brent et al., 1994; Portzky et al., 2005; Wilson et al., 1995) figurent aussi parmi les facteurs déclencheurs du geste suicidaire chez les adolescents, même s'ils peuvent sembler minimes et sans graves conséquences pour un observateur externe (Beautrais, 2001). Or, il appert que les familles desquelles sont issus les jeunes délinquants sont conflictuelles et marquées par une escalade d'altercations (Patterson, DeBaryshe et Ramsey, 1989). C'est la détresse vécue par le jeune à la suite des conflits qu'il est important d'évaluer afin de saisir l'importance qu'ils peuvent revêtir. vécue par l'adolescent (Chagnon, Houle, Marcoux et Renaud, 2007; Gallagher et Dobrin, 2006; Rohde, Seeley et al., 1997; Wasserman et McReynolds, 2006). Être incarcéré pour la première fois constitue un stress important à cause notamment des incertitudes qui y sont associées et des ruptures entraînées avec les réseaux habituels (Roberts et Bender, 2006). En effet, la séparation du milieu naturel place l'adolescent dans une situation de vulnérabilité; or, cette séparation s'inscrit ellemême dans un processus pour pallier une autre situation de risque dans laquelle a pu se placer l'adolescent, en raison de conduites délinquantes (Chagnon et al., 2001; Gallagher et Dobrin, 2006). Au niveau des représentations qu'elle suscite, la prise en charge peut signifier - du point de vue de l'adolescent - l'échec des activités délinquantes et la rupture avec un groupe et des activités qui faisaient jusqu'alors partie de son quotidien et contribuaient à définir son identité. L'adolescent peut se trouver sans repères face à cette inter-

D'UNE PART, L'ANXIÉTÉ PEUT ÊTRE LA MANIFESTATION

DE LA DÉTRESSE DE L'ADOLESCENT ET AINSI ÊTRE EN RELATION AVEC LE DÉSESPOIR LE CONDUISANT AUX COMPORTEMENTS SUICIDAIRES; D'AUTRE PART, ELLE PEUT ÊTRE À CE POINT PARALYSANTE POUR L'ADOLESCENT QU'IL NE PEUT ENTREVOIR D'AUTRE SOLUTION À SON PROBLÈME (DANS CE CAS-CI LES ACTES DÉLINQUANTS ET SES CONSÉQUENCES) QUE LES COMPORTEMENTS SUICIDAIRES.

Dans le cas des adolescents pris en charge par le système judiciaire, l'étude des événements récents pouvant contribuer aux conduites suicidaires mérite une attention particulière. En effet, si ces jeunes connaissent des sanctions judiciaires, plusieurs présentent conjointement d'autres facteurs de stress récents qui ne sont pas nécessairement spécifiques à la situation de délinquance ou de prise en charge, mais qui demeurent des amplificateurs du risque suicidaire (par exemple la perte d'un réseau social existant ou un changement d'école à la suite du placement). En ce sens, dès qu'un adolescent est pris en charge par un établissement de détention pour mineurs ou par le système judiciaire, il est important d'être vigilant quant à ses idéations suicidaires puisque plusieurs changements surviennent. De plus, l'hébergement ajoute en lui-même un stress supplémentaire à la situation déjà difficile diction d'utiliser son mode de fonctionnement habituel. Cette rupture occasionnée par «l'arrêt des conduites délinquantes» peut ainsi représenter une perte importante pour l'adolescent - perte du statut délinquant, perte de liens affectifs, perte de plans d'avenir, perte du réseau social, etc. - et mener ultérieurement à une perte d'espoir chez ceux qui ne seront pas en mesure de trouver d'autres objectifs de vie et/ou de créer de nouveaux liens affectifs. En bref, l'adolescent est à risque lorsqu'il perd ses repères habituels et juge qu'il ne peut poursuivre ses activités délinquantes. Il importe cependant d'insister sur l'impact positif que peut revêtir un arrêt des conduites délinquantes lorsqu'il permet à l'adolescent d'expérimenter de nouvelles façons de faire et de se construire de nouveaux repères. Les intervenants devraient ainsi être particulièrement sensibles à la détresse causée par la perte du statut de 
délinquant et accompagner le jeune susceptible de vouloir découvrir de nouvelles façons de faire.

En somme, les pertes de tout ordre, qu'elles soient réelles ou symboliques, sont susceptibles de causer une grande détresse chez l'adolescent qui les expérimente. Dans le cas des délinquants spécifiquement, il importe donc de ne pas perdre de vue la rupture relationnelle que peut revêtir le fait d'être pris en charge à l'extérieur de son milieu naturel et la rupture avec le mode de vie habituel entraînée par la sanction judiciaire.

\section{Psychopathologie}

Les recherches s'intéressant aux personnes décédées par suicide indiquent qu'environ $90 \%$ présentaient au moins un problème de santé mentale au moment de leur décès (Beautrais, 2000, 2003a; Psychiatry, 2001; Rohde, Mace et al., 1997). Tant les troubles intériorisés qu'externalisés représentent des facteurs de risque de comportements suicidaires, chez les adolescents de la population générale (Portzky et al., 2005 ; Sourander, Helstelä, Haavisto et Bergroth, 2001) et chez ceux incarcérés (Chowanec, Josephson, Coleman et Davis, 1991) ou hébergés en institution (Hukkanen, Sourander et Bergroth, 2003).

Troubles de l'humeur, troubles anxieux, troubles de la personnalité, troubles d'abus de substances et troubles des conduites (Battle et al., 1993 ; Beautrais, 2000; Daigle et Côté, 2006; Dembo et al., 1990; Esposito et Clum, 2002 ; Harris et Lennings, 1993 ; Howard et al., 2003; Penn et al., 2003; Robert Plutchik, Van Praag et Conte, 1989; Putnins, 2005 ; Renaud, Berlim, McGirr, Tousignant et Turecki, 2008; Renaud et al., 1999) sont spécifiquement associés aux comportements suicidaires. En outre, plus le nombre de diagnostics est élevé (troubles concomittants), plus le risque de suicide augmente (Beautrais, 2000, 2003a ; Beautrais, Joyce et Mulder, 1996; Renaud et al., 2008). Environ le tiers des adolescents en centre d'évaluation judiciaire présenteraient au moins un trouble psychiatrique (McReynolds et al., 2008; Nolen et al., 2008). Les adolescents délinquants qui rapportent des tentatives de suicide antérieures ou des idéations suicidaires actuelles présentent un profil plus lourd au niveau de la psychopathologie que ceux qui ne sont pas suicidaires (Ruchkin, Schwab-Stone, Koposov, Vermeiren et King, 2003). Bien que les problèmes de santé mentale ne représentent pas des facteurs de risque qui leur soient spécifiques, la délinquance et la prise en charge qui en résulte peuvent contribuer à l'établissement de certains troubles (anxiété et dépression) et à l'aggravation de ceux existant déjà (Goldstein, Olubadewo, Redding et Lexcen, 2005), dans une dynamique où un facteur de risque en alimente un autre.

Les troubles et symptômes dépressifs sont reconnus comme les troubles les plus souvent associés aux comportements suicidaires chez les adolescents (Apter, Bleich, Plutchik, Mendelsohn et al., 1988; Beautrais, 2000, 2003 a, 2003 b; Connor et Rueter, 2006; Esposito et Clum, 2002 ; Fergusson, Beautrais et Horwood, 2003; Flouri et Buchanan, 2002; Fombonne, 1998; Psychiatry, 2001; Renaud, Chagnon, Turecki et Marquette, 2005; Thompson, Ho et Kingree, 2007). L'épisode dépressif majeur constitue un facteur de risque encore plus important quand il est combiné à un trouble des conduites (Fombonne, Wostear, Cooper, Harrington et Rutter, 2001). Or, la dépression est le trouble le plus fréquent chez les jeunes délinquants (N.E.S. Goldstein et al., 2005, 2005) [en excluant le trouble des conduite, puisque ce dernier concernerait environ $80 \%$ des jeunes incarcérés (Domalanta et al., 2003)], illustrant ainsi leur vulnérabilité. Plus spécifiquement, chez des adolescents présentant des troubles des conduites, lorsque des symptômes dépressifs s'ajoutent à la problématique, les risques de comportements suicidaires augmentent de façon importante (Battle et al., 1993; Davis et al., 1991; Harris et Lennings, 1993 ; Hayes, 2005 ; Kempton et Forehand, 1992; Langhinrichsen-Rohling et al., 2004; Penn et al., 2003; Renaud et al., 2008; Roberts et Bender, 2006; Rohde, Mace et al., 1997 ; Sanislow, Grilo, Fehon, Axelrod et McGlashan, 2003; Wasserman et McReynolds, 2006). Selon les résultats qu'ils ont obtenus, LanghinrichsenRohling et al. (2004) expliquent que les jeunes ayant eu une interaction avec le système de justice sont à la fois plus à risque de présenter des symptômes dépressifs et ont une plus grande propension au suicide. Ces résultats permettent de comprendre comment une situation - l'interaction avec le système de justice dans ce cas-ci - peut entraîner des affects de perte d'espoir et de découragement, pouvant mener à des comportements suicidaires. À l'inverse, la dépression et l'anxiété peuvent être associées au développement du trouble des conduites (Holmes, Slaughter et Kashani, 2001). Ainsi, les problématiques de délinquance et de désespoir tendent à s'influencer réciproquement et à exacerber de part et d'autre le risque de suicide chez ces adolescents.

Avoir un trouble anxieux amplifie le risque suicidaire chez les adolescents (Beautrais, 2003a; Brent et al., 1993 ;
Connor et Rueter, 2006; Esposito et Clum, 2002; Vermeiren et al., 2003). Il en est de même pour les adolescents délinquants ou hébergés en centres de réadaptation (Hukkanen et al., 2003; Penn et al., 2003 ; Rohde, Seeley et al., 1997). Puisque l'anxiété est plus difficile à évaluer que les comportements antisociaux, les intervenants doivent se montrer d'autant plus vigilants. Dans le cas des jeunes délinquants, le risque élevé de présenter de l'anxiété peut résulter des actes antisociaux et délinquants proprement dits, eux-mêmes générateurs de stress et d'anxiété. De même en est-il des conséquences disciplinaires et judiciaires. L'anxiété comme facteur amplifiant le risque suicidaire peut être interprétée et comprise selon deux perspectives cliniques. D'une part, l'anxiété peut être la manifestation de la détresse de l'adolescent et ainsi être en relation avec le désespoir le conduisant aux comportements suicidaires; d'autre part, elle peut être à ce point paralysante pour l'adolescent qu'il ne peut entrevoir d'autre solution à son problème (dans ce cas-ci les actes délinquants et ses conséquences) que les comportements suicidaires.

Bien que les troubles concomitants soient fréquents chez les jeunes délinquants ou chez ceux pris en charge par le système de justice pénale, la plupart de ces jeunes n'auraient néanmoins jamais reçu de diagnostic psychiatrique antérieur à leur prise en charge. Quarante-trois pourcent des jeunes garçons incarcérés présentaient un trouble d'abus d'alcool et $27 \%$ un trouble d'abus de substances (Domalanta et al., 2003). Les troubles les plus fréquemment associés pour cette population seraient ainsi les troubles dépressifs en combinaison avec l'abus de drogues et/ou d'alcool (Domalanta et al., 2003).

\section{Abus de substances}

L'abus de substances et/ou d'alcool est associé aux comportements suicidaires chez les adolescents de la population générale et chez ceux présentant des troubles des conduites (Flouri et Buchanan, 2002; Perkins et Hartless, 2002 ; Putnins, 2005). Une étude (Kotila et Lönnqvist, 1988) révèle que $53 \%$ des garçons et $40 \%$ des filles avaient consommé de l'alcool au moment de leur tentative de suicide. La consommation de substances combinée à d'autres troubles représenterait un risque encore plus grand de comportements suicidaires (Brent et al., 1993). Une large part des adolescents délinquants affiche ce facteur de risque (DeMatteo et Marczyk, 2005 ; N.E.S. Goldstein et al., 2005).

Chagnon et al. (2001) rapportent que les adolescents hébergés en centres de réadaptation et présentant des comportements 
suicidaires avaient consommé de l'alcool plus fréquemment au cours de la dernière année que ceux ne présentant pas de tels comportements. En outre, l'intoxication aux drogues peut être un facteur précipitant du geste suicidaire pour $17 \%$ à $51 \%$ des adolescents décédés par suicide dans les établissements (Chagnon et al., 2001). La consommation de substances peut agir en tant que déclencheur du geste suicidaire en diminuant les inhibitions et augmentant l'impulsivité. De même, la consommation de drogue peut avoir un effet dépresseur et favoriser les idéations suicidaires qui précèdent les comportements (N.E.S. Goldstein et al., 2005). Dans l'étude de Hayes (2004), 87,9\% des adolescents décédés par suicide lors de leur détention présentaient une histoire d'abus de substances. Pour d'autres (Howard et al., 2003), ce sont plutôt $26 \%$ des cas de suicide chez les adolescents en détention qui leur sont associés. Au-delà des divergences, l'abus de substances est très prévalent chez les adolescents qui se suicident en détention. Dans une étude portant sur les adolescents incarcérés (Domalanta et al., 2003), 43\% des garçons et $36 \%$ des filles avaient un problème d'abus d'alcool; $27 \%$ des garçons et des filles avaient un problème d'abus de substances. De plus, l'abus de substances est fortement associé à la délinquance (N.E.S. Goldstein et al., 2005; Hart, O'Toole, Price-Sharps et Shaffer, 2007). Des associations similaires ont été établies entre l'abus de substances et les tentatives de suicide dans cette population (Morris et al., 1995). Plus spécifiquement, l'abus de substances et l'intoxication peuvent interférer avec l'instauration de mécanismes de coping (adaptation) efficaces et augmenter l'exposition aux situations difficiles, contribuant indirectement au risque suicidaire (Ruchkin et al., 2003).

\section{Traits de la personnalité}

Les traits de la personnalité se définissent progressivement à partir de l'enfance; il est généralement admis qu'ils sont le fruit d'un processus complexe marqué par l'imbrication des caractéristiques personnelles et du tempérament de l'enfant.

Les caractéristiques personnelles des adolescents délinquants les mettent plus à risque de présenter des comportements suicidaires (Putnins, 2005; Roberts et Bender, 2006). Plus spécifiquement, dans les études réalisées auprès d'adolescents de la population générale, l'hostilité (Connor et Rueter, 2006), l'irritabilité (Beautrais, 2003a; Conner, Meldrum, Wieczorek, Duberstein et Welte, 2004), la recherche de nouveauté (Beautrais, 2003a; Fergusson et al., 2003), la témérité, les comportements de prise de risque
(Beautrais, 2003a; Vermeiren et al., 2003) et les comportements violents (Apter et al., 1988; Beautrais, 2003a ; Cleary, 2000; Marttunen et Pelkonen, 2000 ; Psychiatry, 2001 ; Zlotnick, Wolfsdorf, Johnson et Spirito, 2003) sont associés au risque de comportements suicidaires. Or, ces mêmes caractéristiques sont utilisées pour décrire les adolescents délinquants et présentant des troubles des conduites (Diagnostic and Statistical Manual of Mental Disorders, 1994) ou pris en charge par le système judiciaire. Elles constituent des facteurs personnels chez les adolescents délinquants car elles contribuent à la fois à la problématique délinquante et amplifient le risque suicidaire. Dans une recension des écrits portant sur le suicide chez les adolescents de la population générale, l'introversion, les traits de personnalité de type passif, dépendant, hystérique et obsessionnel ont été identifiés comme des facteurs prédisposant aux conduites suicidaires (Beautrais, 2003a); il s'agit donc de facteurs de risque non spécifiques aux adolescents délinquants.

L'utilisation de la violence et de l'agressivité dès le jeune âge constitue une caractéristique importante chez les adolescents délinquants (Christoffersen et al., 2007 ; N.E.S. Goldstein et al., 2005 ; Lahey, Waldman et McBurnett, 1999), puisqu'il s'agit également d'éléments ayant généralement contribué à la prise en charge par le système judiciaire. Ces jeunes devenus délinquants ont été décrits dès l'enfance comme ayant un tempérament «difficile», négatifs, anxieux, irritables, colériques, agressifs et impulsifs (Holmes et al., 2001). L'association entre le risque de violence envers autrui et la présence de comportements suicidaires a été étudiée; un individu violent aurait plus de risque de commettre des gestes suicidaires (Apter, Plutchik, Sevy, Korn et al., 1989; Apter, Plutchik et Van Praag, 1993; Chagnon et al., 2001; Daigle et Côté, 2006 ; Horesh et al., 1997; Plutchik, 1995 ; Plutchik, 2000 ; Plutchik, Botsis et Van Praag, 1995; Plutchik et Van Praag, 1990). L'association inverse serait aussi vraie: les adolescents suicidaires démontrent plus de violence envers les autres que ceux qui ne le sont pas (Zlotnick et al., 2003), ils présentent aussi plus de problèmes avec la police (Flouri et Buchanan, 2002). Les comportements violents contribuent au risque suicidaire, sans qu'il ne s'agisse d'un effet indirect de la dépression (Apter, Gothelf, Orbach, Weizman et al., 1995). Ces auteurs (Apter et al., 1995) soutiennent que les jeunes présentant des troubles des conduites seraient plus susceptibles d'avoir des comportements suicidaires impulsifs plutôt qu'un désir de mourir planifié, associé à la dépression majeure.

Des indices très précis, comme avoir été impliqué dans une bagarre au cours de la dernière année ou en avoir été l'instigateur (Evans, Marte, Betts et Silliman, 2001; King et al., 2001), peuvent constituer des indicateurs d'un risque accru de comportement suicidaire. Tant l'agressivité verbale que la violence physique y sont associées (Beautrais, 2003a). Les recherches auprès d'adolescents délinquants établissent la même association entre agressivité et suicide (Brent et Bridge, 2007; Chagnon et al., 2001; Chowanec et al., 1991; Hukkanen et al., 2003; Wasserman et McReynolds, 2006). Plutchik (Plutchik et al., 1989) a étudié l'association entre agressivité dirigée à l'extérieur de soi (violence envers autrui) et contre soi (comportements suicidaires) chez des adultes incarcérés. Ces deux formes d'agressivité (vers l'extérieur et envers soi-même) posséderaient des caractéristiques communes, mais il serait tout de même possible de distinguer les modes de recours à l'une ou à l'autre.

L'impulsivité représente également une caractéristique associée au risque de violence et au risque de suicide (Apter et al., 1993; Battle et al., 1993; Chagnon et al., 2001; Conner et al., 2004; DeMatteo et Marczyk, 2005). Alors que la colère ne le permettrait pas, l'impulsivité distinguerait les patients suicidaires des patients non suicidaires (Hoge, Andrews et Leschied, 1992). Les patients ayant commis une tentative ou présentant des idéations suicidaires démontreraient en effet une plus grande impulsivité que ceux ne présentant pas ces comportements. L'impulsivité représenterait une caractéristique commune entre troubles externalisés et comportements suicidaires (Hills, Cox, McWilliams et Sareen, 2005). Dans les recherches spécifiques aux adolescents délinquants, l'impulsivité apparaît comme un facteur de risque important des conduites suicidaires (Brent et Bridge, 2007; Chagnon et al., 2001; Roberts et Bender, 2006 ; Rohde, Mace et al., 1997 ; Sanislow et al., 2003); ces adolescents impulsifs s'accordent moins de temps pour réfléchir à leurs actions, pouvant les conduire à commettre des gestes irrémédiables, tels les comportements suicidaires.

L'estime de soi est une caractéristique pouvant modérer les comportements suicidaires. Plus spécifiquement, des déficits à ce niveau pourraient jouer un rôle important dans les comportements suicidaires à l'adolescence (Overholser, Adams, Lehnert et Brinkman, 1995). Ainsi, présenter une faible estime de soi (Breton et al., 2002; Overholser et al., 1995 ; Psychiatry, 2001) et se sentir responsable des événements 
négatifs (Psychiatry, 2001) augmenteraient le pessimisme et le désespoir de l'adolescent au regard de son avenir (Overholser et al., 1995). En combinaison avec l'impulsivité, il pourrait s'agir d'un cocktail favorisant l'émergence de comportements suicidaires.

Au-delà d'un risque accru par une pauvre estime de soi, l'Enquête sociale et de santé auprès des enfants et des adolescents québécois 1999 (Breton et al., 2002) a montré que les adolescents avec une meilleure estime de soi présentaient moins d'idées suicidaires sérieuses. D'autres études portant sur les adolescents ont également mis de l'avant ce facteur de protection pour les comportements suicidaires (Beautrais, 2000, 2003a ; Beautrais, Joyce et Mulder, 1999; Fergusson et al., 2003 ; Pronovost, Leclerc et Dumont, 2003; Psychiatry, 2001; Thompson et al., 2007).

Les adolescents délinquants sont susceptibles de présenter une estime d'eux-mêmes diminuée en raison des conséquences négatives entraînées par les gestes qu'ils ont posés et qui les ont menés à être pris en charge. En effet, une large part de la réadaptation dont ils bénéficient vise à favoriser une prise de conscience chez eux de la gravité des gestes qu'ils ont commis. Bien qu'il soit souhaitable que ces adolescents en viennent à être en mesure de mieux évaluer les conséquences de leurs comportements, les intervenants doivent demeurer vigilants dans ces périodes où pourraient surgir des sentiments de culpabilité ou de perte d'estime de soi.

\section{Coping}

Les habiletés de coping constituent un facteur de protection ou de risque au regard des conduites suicidaires. La capacité de s'adapter aux situations difficiles et de présenter de bonnes habiletés de résolution de problèmes protégeraient des conduites suicidaires (Beautrais, 2000, 2003a; Chagnon et al., 2001 ; Esposito et Clum, 2002). De même, avoir un sentiment de maîtrise sur son environnement, pouvoir considérer les situations difficiles selon diverses perspectives, posséder des habiletés de résolution de problème et de conflits, contrôler sa colère et avoir une pensée critique protégerait des comportements antisociaux (DeMatteo et Marczyk, 2005). Faire preuve de contrôle de son impulsivité constitue un facteur de protection au regard des comportements suicidaires; or, les jeunes en centres de réadaptation contrôlent moins leurs conduites impulsives que les adolescents qui n'y résident pas (Pronovost et al., 2003). Les adolescents détenus ayant une histoire de tentative de suicide présentent des déficits de résolution de problème comparativement à ceux n'ayant jamais commis de tentatives (Speckens et Hawton, 2005). À l'inverse, des lacunes ou un manque de confiance dans ses habiletés de résolution de problèmes (Esposito et Clum, 2002; Psychiatry, 2001 ; Speckens et Hawton, 2005), une moins bonne gestion de la colère et de la tristesse ainsi que la réaction aux événements difficiles par le blâme ou l'agressivité seraient des facteurs de risque de suicide (Chagnon et al., 2007 ; Chagnon et al., 2001). Puisque le contrôle de soi des adolescents délinquants a été identifié comme généralement déficient (Marcotte, Marcotte et Bouffard, 2002), ils pourraient présenter un risque accru de suicide en lien avec l'impulsivité qu'ils démontrent.

Les adolescents hébergés en centres de détention présenteraient des mécanismes d'adaptation moins efficaces aux situations conflictuelles, se plaçant ainsi plus à risque de comportements suicidaires (Chagnon et al., 2001). Les intervenants doivent être attentifs lorsque les jeunes dont ils ont la responsabilité expérimentent des situations difficiles ou stressantes. Ces derniers peuvent en effet se sentir démunis et ne pas savoir comment réagir, se mettant ainsi plus à risque de répondre de façon inadaptée, voire de présenter des comportements suicidaires.

\section{QUELQUES FACTEURS}

MICROSYSTÉMIQUES

Dans cette section seront abordés deux principaux agents de soutien et de socialisation à l'adolescence: la famille et les parents ainsi que le groupe de pairs. La famille et les amis peuvent exercer une influence considérable sur les sentiments et pensées des adolescents. Plusieurs études démontrent que le soutien social est un facteur de protection important au regard des comportements suicidaires (Battle et al., 1993; Breton et al., 2002; Connor et Rueter, 2006; Flouri et Buchanan, 2002; Pronovost et al., 2003).

\section{Famille / parents}

L'adolescence constitue une période durant laquelle la principale tâche développementale est de se distancier de sa famille et d'investir dans des liens affectifs à l'extérieur du cercle familial; d'où la référence à l'adolescence comme seconde phase de séparation-individuation (Blos, 1967). La famille et les parents demeurent néanmoins fort importants dans la vie de l'adolescent et leur influence est considérable. De même, ils demeurent les premiers agents de socialisation dans l'histoire de l'adolescent.

L'influence de la famille sur les comportements suicidaires des adolescents peut relever de diverses sources. Ainsi, la présence d'histoires de suicides dans la famille prédisposerait les adolescents à présenter eux-mêmes des conduites suicidaires (Beautrais, 2000, 2001, 2003a; Brent et al., 1996; Fergusson et al., 2003 ; Portzky et al., 2005; Psychiatry, 2001; Renaud et al., 2008). De plus, la psychopathologie des parents pourrait constituer un facteur de risque (Brent, Baugher, Bridge, Chen et Chiappetta, 1999; Flouri et Buchanan, 2002; King et al., 2001; Portzky et al., 2005). Des parents qui abusent de substances, des mères déprimées, des pères antisociaux, violents et abusifs mettraient les adolescents plus à risque de présenter des comportements suicidaires (Beautrais, 2000; Brent et al., 1996; Brent et Bridge, 2007 ; Brent et al., 1994; Psychiatry, 2001; Renaud et al., 1999), tout comme des parents ayant des difficultés légales (Brent et al., 1994) ou impliqués dans le milieu criminel (Brent et Bridge, 2007) entraîneraient des risques plus élevés de comportements suicidaires et de délinquance (Alltucker et al., 2006; Lahey et al., 1999) chez leurs adolescents. Les enfants ayant grandi dans des familles caractérisées par la violence familiale, où les parents abusent de substances, présentent des troubles psychiatriques et n'occupent pas d'emploi stable seraient plus à risque de développer des problématiques délinquantes (Christoffersen et al., 2007 ; DeMatteo et Marczyk, 2005). Le fait de vivre dans une famille où les parents sont séparés augmenterait le risque de comportements suicidaires à l'adolescence (Flouri, 2005 ; Flouri et Buchanan, 2002).

Les facteurs de risque découlant des parents (psychopathologie, conduites suicidaires, criminalité) peuvent compromettre les habiletés parentales et ne pas permettre à ces derniers d'agir en tant qu'agents de référence stables auprès de leurs enfants, contribuant ainsi à la fois à leur risque suicidaire et de délinquance. La supervision et le soutien émotionnel par les parents sont essentiels à l'adolescence (Flouri, 2005 ; Flouri et Buchanan, 2002 ; Kidd et al., 2006; Perkins et Hartless, 2002). Ainsi, des attentes parentales disproportionnées (trop ou trop peu élevées), favoriseraient les comportements suicidaires des adolescents (Beautrais, 2000). De même, un manque de supervision de la part des parents augmenterait à la fois le risque suicidaire (King et al., 2001) et le risque de délinquance (Krisberg et Wolf, 2005 ; Lahey et al., 1999).

Auprès d'adolescents incarcérés, un contrôle de la part du père sans démonstration d'affection serait lié à plus d'idéations et de tentatives de suicide, mènerait à une plus faible estime de soi et à des sentiments de désespoir (McGarvey, Kryzhanovskaya, 
Koopman, Waite et Canterbury, 1999). Les adolescents délinquants présentant un profil suicidaire perçoivent leurs parents comme moins chaleureux et plus rejetants (Ruchkin et al., 2003). En plus de contribuer au risque suicidaire, les pratiques parentales et le climat familial sont fortement associés au développement des comportements délinquants (Butler, Fearon, Atkinson et Parker, 2007; Farrington, 1994 ; Patterson et al., 1989). En effet, les enfants issus de familles instables et conflictuelles présentent plus de risque de délinquance que ceux issus de familles unies et paisibles où les parents offrent une supervision adéquate et constante (Patterson et al., 1989).

Comme c'est le cas pour les conduites suicidaires, le milieu familial a un rôle à jouer dans le développement de la délinquance. Ainsi, avoir des parents ou des membres de sa fratrie impliqués dans le milieu criminel ou en prison constitue un facteur de risque de délinquance (Farrington, 1994). Ces problèmes de la part des parents rendraient les adolescents plus à risque parce que le réseau social familial est affaibli (Brent et al., 1994). De plus, grandir dans un milieu où la délinquance est le principal mode de fonctionnement pourrait susciter le recours à des modes de fonctionnement similaires par les jeunes, à défaut de connaître d'autres façons d'agir. Dans un même ordre d'idées, les familles caractérisées par les conflits familiaux seraient plus à risque de favoriser des comportements délinquants de la part des adolescents (Butler et al., 2007; Patterson et al., 1989). Des conditions familiales difficiles seraient le facteur le plus puissant pour prédire la délinquance persistant à l'âge adulte (Lay et al., 2005). Être témoin de violence conjugale augmenterait le risque suicidaire chez les adolescents (Flouri, 2005).

De bonnes relations familiales peuvent constituer un facteur de protection à l'encontre d'autres circonstances désavantageuses (par ex. provenir de milieu issu de la pauvreté). Les adolescents estimant bénéficier d'un niveau élevé de soutien de la part de leurs parents et de leurs pairs (Breton et al., 2002; Pronovost et al., 2003) présentent moins d'idées suicidaires et sont moins susceptibles d'avoir fait une tentative dans le passé (Flouri et Buchanan, 2002). Les relations parents-enfants chaleureuses, positives, où les parents sont engagés et disponibles émotionnellement protègent des comportements suicidaires chez les adolescents (Battle et al., 1993 ; Connor et Rueter, 2006; Flouri et Buchanan, 2002). Les adolescents ayant des relations positives avec leurs parents et leurs profes- seurs démontrent en général moins de problèmes comportementaux (Crosnoe, Erickson et Dornbusch, 2002; Hart et al., 2007). Le fait de vivre avec au moins un parent constitue un facteur de protection des conduites suicidaires; les jeunes qui ne vivent pas avec au moins un parent biologique présentent un risque accru (Brent et al., 1994; Flouri et Buchanan, 2002; Roberts et Bender, 2006 ; Rohde, Seeley et al., 1997 ; Sourander et al., 2001).

Il est possible de constater que la plupart des adolescents délinquants et pris en charge par le système judiciaire ne bénéficient pas de la protection qui puisse être apportée par de bonnes relations avec les parents. En effet, les parents d'adolescents délinquants peuvent faire usage de pratiques parentales inadéquates, ce qui a pu amplifier la problématique de délinquance; ou encore, être inadéquates en raison des comportements de leurs enfants auxquels ils ne savent comment réagir. Ces influences parentales jouent un rôle très important et peuvent contribuer à l'instauration du risque suicidaire, risque pouvant être exacerbé par une problématique de délinquance s'étant installée parallèlement.

\section{Pairs}

Outre les parents et la famille, les amis sont particulièrement importants à l'adolescence. Le soutien social est un facteur capital, particulièrement lors d'événements de vie stressants : il peut aider un individu à les traverser sans présenter de conduite suicidaire (Battle et al., 1993; Beautrais, 2000, 2003a; Chagnon et al., 2001; Kidd et al., 2006).

Des études s'étant intéressées aux adolescents délinquants ont révélé un réseau social déficient chez ces jeunes (Rohde, Seeley et al., 1997) (DeMatteo et Marczyk, 2005). À cause de leurs comportements hostiles, agressifs et violents, ces jeunes étaient souvent des enfants non populaires à l'école, contribuant au développement de leurs comportements délinquants (N.E.S. Goldstein et al., 2005) et à l'affiliation à des pairs présentant des comportements semblables (Holmes et al., 2001). De plus, le rejet par les pairs peut entraîner désespoir, dépression, idéations et comportements suicidaires (Prinstein, Boergers, Spirito, Little et Grapentine, 2000). L'association à des pairs délinquants augmente le risque de délinquance (Crosnoe et al., 2002; DeMatteo et Marczyk, 2005; Krisberg et Wolf, 2005). Lorsqu'ils font partie d'un gang, ces adolescents présentent plus de comportements suicidaires (Morris et al., 1995 ; Prinstein et al., 2000). Bien que l'affiliation à un groupe de pairs délinquants augmente la vulnérabilité du jeune au sui- cide (Beautrais, 2003a; Fergusson et al., 2003), elle peut dans certains cas constituer le seul réseau de soutien de l'adolescent délinquant. Les intervenants doivent donc demeurer sensibles à l'importance de ce groupe pour le jeune et s'interroger sur les conséquences possibles d'une rupture de lien avec un groupe délinquant: «Est-il plus dommageable pour le jeune de perdre le seul réseau social disponible ou d'avoir comme seul réseau social un groupe délinquant?»

\section{Adultes significatifs non parentaux}

Entretenir des relations positives avec au moins un adulte significatif dans la communauté protégerait des comportements délinquants et violents chez les jeunes qui sont à risque (Hart et al., 2007).

\section{FACTEURS ORGANISATIONNELS \\ ET SOCIAUX}

Les structures organisationnelles, sociales et systémiques dans lesquelles évolue l'individu sont essentielles à cerner pour comprendre des phénomènes tels les comportements suicidaires et la délinquance puisqu'elles régissent et teintent l'influence des facteurs abordés précédemment.

\section{Voisinage et milieu de vie}

Avoir déménagé au cours de la dernière année (Brent et al., 1994), de même que vivre à la ville plutôt qu'à la campagne (Evans et al., 2001), placerait les adolescents plus à risque de gestes suicidaires. Provenir d'un milieu désavantagé sur le plan socioéconomique constituerait un facteur de risque quant aux comportements suicidaires (Beautrais, 2000, 2003a; Brent et Bridge, 2007) et délinquants (DeMatteo et Marczyk, 2005; Farrington, 1994). Cela ne constituerait cependant qu'un facteur de risque indirect. En effet, à cause de difficultés d'ordre économique, les parents peuvent ne pas avoir les ressources nécessaires pour assumer pleinement leur rôle parental (par ex.: heures de travail non régulières, précarité financière ne permettant pas la participation aux activités sportives et culturelles, etc.). Le milieu de vie de l'adolescent peut constituer un risque, notamment si des armes ou d'autres moyens pour tenter de se suicider sont facilement accessibles (Rohde, Seeley et al., 1997 ; Wasserman et McReynolds, 2006). Dans l'évaluation du risque suicidaire, il est donc important de prendre en compte le milieu dans lequel évolue l'adolescent afin de savoir sur quoi il importe d'agir dans l'immédiat. En effet, si un moyen de suicide est à la disposition de l'adolescent, il sera primordial d'intervenir à ce niveau pour réduire le risque de suicide. 


\section{École}

Connaître une expérience scolaire positive pourrait constituer un facteur de protection pour les adolescents (Beautrais, 2003a). En effet, il y a tout lieu de supposer que cela favorise une bonne estime de soi chez le jeune, en plus de fournir un programme quotidien et de mettre l'adolescent en lien avec des personnes (jeunes et adultes) pouvant constituer un réseau positif (Kidd et al., 2006; Kotila et Lönnqvist, 1988). À l'inverse, une faible motivation académique, des attitudes négatives face à l'école et des difficultés scolaires représentent des facteurs de risque de comportements suicidaires (Flouri et Buchanan, 2002; Hart et al., 2007 ; Portzky et al., 2005 ; Psychiatry, 2001). Le fait de quitter l'école et de ne pas atteindre des qualifications minimales placerait les adolescents plus à risque de présenter des comportements suicidaires (Beautrais, 2003a, 2003b; Brent et Bridge, 2007 ; Fergusson et al., 2003 ; Rohde, Seeley et al., 1997).

En ce qui concerne l'impact de l'école sur la délinquance, entretenir des attitudes positives concernant l'importance de l'école, de bonnes relations avec ses professeurs et un bon rendement scolaire protégerait de l'influence négative des amitiés délinquantes (Crosnoe et al., 2002). C'est en ce sens que l'influence des adultes significatifs, notamment les professeurs, peut jouer un rôle clé dans la prévention de la délinquance, tout en favorisant la réussite scolaire et ainsi la valorisation de l'école.

\section{Établissements de détention /}

\section{d'hébergement}

Le fait de vivre dans un établissement pour délinquants juvéniles constituerait en soi un facteur de risque (Memory, 1989; Pena et Caine, 2006; Roberts et Bender, 2006). Certains éléments spécifiques aux structures d'hébergement contribueraient au risque de suicide: des établissements où les portes des chambres sont verrouillées constituent des milieux plus à risque (Gallagher et Dobrin, 2006), tout comme ceux où les jeunes sont en chambres individuelles (Hayes, 2005). Afin d'expliquer ce taux élevé de suicide dans les établissements pour délinquants juvéniles, certains (Howard et al., 2003) ont souligné que le nombre de tentatives de suicide pourrait refléter le niveau de détresse et de difficulté de l'adolescent contrevenant; plus cette détresse est grande, plus le nombre de tentatives serait élevé. La tentative de suicide pourrait être considérée comme le symptôme d'un état de détresse et de désorganisation envahissant. De même, ces institutions hébergent des adolescents qui sont déjà à risque de suicide et de compor- tements suicidaires par les problèmes qu'ils présentent déjà au moment de leur admission (Roberts et Bender, 2006). En effet, il s'agirait ici en quelque sorte d'un «biais de sélection» de l'échantillon à l'étude: puisque les adolescents sont pris en charge par le système judiciaire parce qu'ils présentent des problèmes comportementaux, souvent liés à des expériences difficiles de tout ordre, il est possible de concevoir qu'ils sont déjà à risque de comportements suicidaires (tentatives, menaces et suicide complété) avant même leur admission.

\section{IMPLICATIONS POUR LA PRATIQUE ET LA RECHERCHE}

Cet article avait pour objectif premier d'examiner les liens entre suicide et délinquance. En ce sens, il est possible de constater que des caractéristiques personnelles, telles que l'impulsivité, l'agressivité et l'abus de substances (Brent et Bridge, 2007 ; Brent et al., 1994), contribuent à l'établissement et à la persistance des deux problématiques. Les facteurs menant à la prise en charge par des services de protection pour enfants et adolescents recoupent ceux pouvant conduire aux comportements suicidaires (Farand et al., 2004). Il est ainsi possible d'inclure dans cette catégorie les facteurs touchant le contexte familial tels la désorganisation, le faible soutien social, un climat de violence familiale (Farand et al., 2004). De même, des facteurs individuels peuvent mener un adolescent à être pris en charge et constituent des facteurs de risque suicidaires : l'abus de substances, les troubles du comportement, des traits d'impulsivité et d'agressivité (Farand et al., 2004).

En tant que facteurs associés à la délinquance plus spécifiquement, les bagarres, le port d'armes, la consommation excessive d'alcool et la consommation de drogue sont fréquents chez les délinquants (Patterson et al., 1989) et constituent des facteurs de risque suicidaires puisque ces comportements mettent les jeunes à risque en tant que tels (Brent et Bridge, 2007). De plus, il est démontré que l'accès à une arme combiné à une consommation de drogue et d'alcool augmente les probabilités de passer à l'acte lorsque des idéations suicidaires surgissent, mettant ainsi les jeunes impliqués dans le milieu de la délinquance plus à risque (Brent et Bridge, 2007).

Les conséquences mêmes de la délinquance, à savoir les problèmes légaux et disciplinaires, l'affiliation à des pairs délinquants et des difficultés interpersonnelles sont directement associées au risque suicidaire (Brent et Bridge, 2007 ; Farand et al., 2004) chez ces jeunes et constituent des facteurs de risque qui leur sont spécifiques. Il est très important de garder à l'esprit que l'accumulation des facteurs de risque abordés dans cet article contribue à augmenter le risque de développer la délinquance (DeMatteo et Marczyk, 2005) et de présenter des comportements suicidaires.

LA PRISE EN CHARGE

DE L'ADOLESCENT DÉLINQUANT,

BIEN QU'INDIQUÉE, CONTRIBUE À

AMPLIFIER LE RISQUE SUICIDAIRE

EN OCCASIONNANT DES STRESS

ET DES CHANGEMENTS DANS LE

MODE DE VIE DE L'ADOLESCENT.

\section{Implications pour la pratique}

Diverses études ont pu identifier que les troubles dépressifs tels que la dépression majeure et la dysthymie, de même que les symptômes dépressifs tels que la résignation acquise et le désespoir placent les adolescents délinquants plus à risque de présenter des comportements suicidaires, allant de l'idéation au suicide complété. Il en est de même pour les troubles de comportements et les troubles anxieux. Il importe donc pour les intervenants de porter une attention particulière à la présence de troubles intériorisés chez des adolescents présentant, au premier plan, des troubles extériorisés qui, en raison de leurs manifestations beaucoup plus explosives, peuvent occulter des problématiques plus difficiles à évaluer par un observateur, telles les idéations suicidaires. Ainsi, le danger est que les troubles extériorisés masquent des problématiques intériorisées restant ainsi non dépistées et non traitées. Les troubles extériorisés peuvent occuper toute la place par leurs manifestations explosives, rendant ainsi difficile d'évaluer les troubles intériorisés qui peuvent se cacher derrière et amener les intervenants à concentrer leurs interventions à ce niveau, négligeant de s'adresser à la détresse qui demeure muette.

En ce qui concerne les événements difficiles et les sources de stress, la vigilance est de mise quant à la détresse qu'ils engendrent chez l'adolescent. Qu'il s'agisse de conflits familiaux, d'une rupture amoureuse ou de la prise en charge par le système judiciaire, c'est l'importance accordée à cet événement par l'adolescent qui modulera sa vulnérabilité et sa susceptibilité à présenter des conduites suicidaires. 
Des événements de vie spécifiques à la situation de la prise en charge des jeunes délinquants peuvent augmenter sa vulnérabilité. Les ruptures affectives entraînées par la prise en charge sont spécifiques au cas des adolescents délinquants. De même, certains moments clés dans le processus judiciaire peuvent créer une plus grande détresse et augmenter le risque de suicide, notamment le moment de la comparution ou les premiers jours de la prise en charge. L'intervenant averti sera donc à l'affût des indications d'impuissance ou de désespoir suivant des événements difficiles pour le jeune contrevenant.

La prise en charge de l'adolescent délinquant, bien qu'indiquée, contribue à amplifier le risque suicidaire en occasionnant des stress et des changements dans le mode de vie de l'adolescent. De plus, des éléments organisationnels et relevant de l'environnement physique dans lequel a lieu l'hébergement peuvent s'ajouter aux amplificateurs du risque suicidaire (portes verrouillées et chambres individuelles). La prise en charge par des établissements pour mineurs constitue ici un amplificateur du risque suicidaire associé et modulé par la situation de délinquance. L'hébergement peut aussi se présenter comme une façon de protéger l'adolescent contre des tendances suicidaires. Dans un tel cas, il est primordial que les intervenants soient sensibles aux éléments, dans le contexte de l'hébergement, qui pourraient atténuer ce risque plutôt que de l'accentuer. Les intervenants devraient ainsi être particulièrement sensibles à la détresse causée par la perte du statut de délinquant (l'adolescent qui est accusé et pris en charge ne peut plus endosser le rôle de «délinquant» qu'il avait à l'extérieur) et accompagner le jeune susceptible de vouloir découvrir de nouvelles façons de faire.

Il est essentiel de favoriser les liens entre les jeunes à risque et des modèles adultes positifs étant donné que de tels liens pourraient prévenir à la fois suicide et délinquance. Que ce soit à l'école ou dans d'autres milieux, des adultes significatifs peuvent pallier les difficultés éprouvées par les parents dans l'éducation de leurs adolescents. De même, l'adolescence constitue une période où le jeune doit investir des relations hors du contexte familial; il y a tout lieu d'espérer que ces relations soient positives et l'orientent vers la bonne voie.

Les intervenants impliqués auprès de ces jeunes devraient de même être particulièrement attentifs à des déficits de l'estime de soi des adolescents délinquants qui pourraient indiquer un risque accru de comportements suicidaires. Par la prise en charge, les adolescents sont susceptibles de connaître une baisse de l'estime de soi au cours du processus de reconnaissance des torts qu'ils ont causés.

\section{Implications pour la recherche}

La recension des écrits a permis de confirmer que la délinquance et les comportements suicidaires partagent des caractéristiques communes. Plusieurs questions surgissent à la suite de l'examen de ces facteurs de risque communs. Il est primordial de comprendre ce qui distingue les adolescents délinquants ayant des comportements suicidaires des adolescents délinquants ne présentant pas de tels comportements. Une autre question de recherche importante est de comprendre en quoi la délinquance est en soi un facteur de risque pour les comportements suicidaires à l'adolescence (quel est le rôle de la délinquance en tant que telle dans le risque de suicide vs le rôle des facteurs de risque de la délinquance). Il importe également de mieux comprendre les facteurs spécifiques à cibler pour la prévention et les traitements des jeunes à risque. Enfin, la connaissance de l'épidémiologie des différents comportements (tentatives, menaces et suicides complétés) au sein de la population délinquante juvénile demeure à développer, notamment au Québec où très peu d'études ont examiné les comportements suicidaires présentés par les délinquants juvéniles.

\section{Bibliographie}

ABRAM, K.M., J.Y. CHOE, J.J. WASHBURN, L.A. TEPLIN, D.C. KING et M.K. DULCAN (2008). "Suicidal ideation and behaviors among youths in juvenile detention ", Journal of the American Academy of Child and Adolescent Psychiatry, vol. 47, nº 3, p. 291300 .

ALESSI, N.E., M. MCMANUS, A. BRICKMAN et L. GRAPENTINE (1984). "Suicidal behavior among serious juvenile offenders", American Journal of Psychiatry, vol. 141, $\mathrm{n}^{\circ} 2$, p. 286-287.

ALLTUCKER, K.W., M. BULLIS, D. CLOSE et P. YOVANOFF (2006). "Different pathways to juvenile delinquency: Characteristics of early and late starters in a sample of previously incarcerated youth ", Journal of Child and Family Studies, vol. 15, n ${ }^{\circ} 4$, p. 479-492.

APTER, A., A. BLEICH, R. PLUTCHIK, S. MENDELSOHN et al. (1988). "Suicidal behavior, depression, and conduct disorder in hospitalized adolescents », Journal of the American Academy of Child and Adolescent Psychiatry, vol. 27, n 6, p. 696-699.

APTER, A., D. GOTHELF, I. ORBACH, R. WEIZMAN et al. (1995). "Correlation of suicidal and violent behavior in different diagnostic categories in hospitalized adolescent patients ", Journal of the American Academy of Child and Adolescent Psychiatry, vol. 34, $\mathrm{n}^{\circ}$ 7, p. 912-918.
APTER, A., R. PLUTCHIK, S. SEVY, M.L. KORN et al. (1989). "Defense mechanisms in risk of suicide and risk of violence », American Journal of Psychiatry, vol. 146, $\mathrm{n}^{\circ} 8$, p. 1027-1031.

APTER, A., R. PLUTCHIK et H.M. VAN PRAAG (1993). «Anxiety, impulsivity and depressed mood in relation to suicidal and violent behavior ", Acta Psychiatrica Scandinavica, vol. 87, $\mathrm{n}^{\circ}$ 1, p. 1-5.

BATTLE, A.O., M.V. BATTLE et E.A. TOLLEY (1993). "Potential for suicide and aggression in delinquents at Juvenile Court in a southern city", Suicide and Life-Threatening Behavior, vol. 23, n ${ }^{\circ} 3$, p. 230-244.

BEAUTRAIS, A.L. (2000). «Risk factors for suicide and attempted suicide among young people ", Australian and New Zealand Journal of Psychiatry, vol. 34, $\mathrm{n}^{\circ}$ 3, p. 420-436.

BEAUTRAIS, A.L. (2001). "Child and young adolescent suicide in New Zealand", Australian and New Zealand Journal of Psychiatry, vol. 35, n ${ }^{\circ}$ 5, p. 647-653.

BEAUTRAIS, A.L. (2002). "Gender issues in youth suicidal behaviour ", Emergency Medicine, $\mathrm{n}^{\circ}$ 14, p. 35-42.

BEAUTRAIS, A.L. (2003a). "Life course factors associated with suicidal behaviors in young people", American Behavioral Scientist. Special Issue: Suicide in Youth, vol. 46, $\mathrm{n}^{\circ}$ 9, p. 1137-1156.

BEAUTRAIS, A.L. (2003b). «Suicide and serious suicide attempts in youth: A multiple-group comparison study ", American Journal of Psychiatry, vol. 160, n ${ }^{\circ}$ 6, p. 1093 1099.

BEAUTRAIS, A.L., P.R. JOYCE et R.T. MULDER (1996). "Risk factors for serious suicide attempts among youths aged 13 through 24 years ", Journal of the American Academy of Child and Adolescent Psychiatry, vol. 35, n 9 , p. 1174-1182.

BEAUTRAIS, A.L., P.R. JOYCE et R.T. MULDER (1999). "Personality traits and cognitive styles as risk factors for serious suicide attempts among young people », Suicide and Life-Threatening Behavior, vol. 29, $\mathrm{n}^{\mathrm{0}}$ 1, p. 37-47.

BLOS, P. (1967). «The second individuation process of adolescence», Psychoanalytic Study of the Child, $\mathrm{n}^{\circ} 22$, p. 162-186.

BRENT, D.A., M. BAUGHER, J. BRIDGE, T. CHEN et L. CHIAPPETTA (1999). «Ageand sex-related risk factors for adolescent suicide ", Journal of the American Academy of Child and Adolescent Psychiatry, vol. 38, $\mathrm{n}^{\circ} 12$, p. 1497-1505.

BRENT, D.A., J. BRIDGE, B.A. JOHNSON et J. CONNOLLY (1996). "Suicidal behavior runs in families: A controlled family study of adolescent suicide victims ", Archives of General Psychiatry, vol. 53, $\mathrm{n}^{\circ} 12$, p. $1145-1152$.

BRENT, D.A. et J.A. BRIDGE (2007). «Delinquent accounts: Does delinquency account for suicidal behavior? ", Journal of Adolescent Health, vol. 40, no 3, p. 204205. 
BRENT, D.A., J.A. PERPER, G. MORITZ, C. ALLMAN et al. (1993). «Psychiatric risk factors for adolescent suicide: A case-control study ", Journal of the American Academy of Child and Adolescent Psychiatry, vol. 32, $\mathrm{n}^{\circ} 3$, p. 521-529.

BRENT, D.A., J.A.P ERPER, G. MORITZ, L. LIOTUS et al. (1994). "Familial risk factors for adolescent suicide: A case-control study», Acta Psychiatrica Scandinavica, vol. $89, \mathrm{n}^{\circ} 1$, p. 52-58.

BRETON, J.J., G. LÉGARÉ, J. LAVERDURE et Y. D'AMOURS (2002). Santé mentale, Gouvernement du Québec.

BUTLER, S., P. FEARON, L. ATKINSON et K. PARKER (2007). "Testing an interactive model of symptom severity in conduct disordered youth: Family relationships, antisocial cognitions, and social-contextual risk ", Criminal Justice and Behavior, vol. 34, $\mathrm{n}^{\circ} 6$, p. 721-738.

CHAGNON, F. (2000). Les événements stressants, les mécanismes d'adaptation et le suicide chez les adolescents admis en centres de réadaptation, Montréal, Université du Québec à Montréal, UQAM 206.

CHAGNON, F., J. HOULE, I. MARCOUX et J. RENAUD (2007). «Control-group study of an intervention training program for youth suicide prevention ", Suicide and Life-Threatening Behavior, vol. 37, n 2, p. 135-144.

CHAGNON, F., J. RENAUD et L. FARAND (2001). "Suicide et délinquance juvénile: phénomènes distincts ou manifestations d'une même problématique? ", Criminologie, vol. 34, $\mathrm{n}^{\circ}$ 2, p. 85-102.

CHAPMAN, J.F. et J.D. FORD (2008). "Relationships between suicide risk, traumatic experiences, and substance use among juvenile detainees ", Archives of Suicide Research, $\mathrm{n}^{\circ} 12$, p. 50-61.

CHOWANEC, G.D., A.M. JOSEPHSON, C. COLEMAN et H. DAVIS (1991). "Selfharming behavior in incarcerated male delinquent adolescents », Journal of the American Academy of Child and Adolescent Psychiatry, vol. 30, $\mathrm{n}^{\circ}$ 2, p. 202-207.

CHRISTOFFERSEN, M.N., K. SOOTHILL et B. FRANCIS (2007). "Violent life events and social disadvantage: A systematic study of the social background of various kinds of lethal violence, other violent crime, suicide, and suicide attempts ", Journal of Scandinavian Studies in Criminology and Crime Prevention, vol. 8, n 2, p. 157-184.

CLEARY, S.D. (2000). "Adolescent victimization and associated suicidal and violent behaviors", Adolescence, vol. 35, n 140, p. 671-682.

CONNER, K.R., S. MELDRUM, W.F. WIECZOREK, P.R. DUBERSTEIN et J.W. WELTE (2004). "The association of irritability and impulsivity with suicidal ideation among 15- to 20-year-old males », Suicide and LifeThreatening Behavior, vol. 34, no 4, p. 363373.

CONNOR, J.J. et M.A. RUETER (2006). "Parent-child relationships as systems of support or risk for adolescent suicidality», Journal of Family Psychology, vol. 20, no 1 , p. $143-155$.
CROSNOE, R., K.G. ERICKSON et S.M. DORNBUSCH (2002). "Protective functions of family relationships and school factors on the deviant behavior of adolescent boys and girls: Reducing the impact of risky friendships ", Youth et Society, vol. 33, n 4, p. 515-544.

DAIGLE, M.S. et G. CÔTÉ (2006). «Nonfatal suicide-related behavior among inmates: Testing for gender and type differences ", Suicide and Life-Threatening Behavior, vol. 36, no 6, p. 670-681.

DAVIS, D.L., G.J. BEAN, J.E. SCHUMACHER et T.L. STRINGER (1991). «Prevalence of emotional disorders in a juvenile justice institutional population », American Journal of Forensic Psychology, vol. 9, $\mathrm{n}^{\circ} 1$, p. 5-17.

DEMATTEO, D. et G. MARCZYK (2005). «Risk factors, protective factors, and the prevention of antisocial behavior among juveniles ", dans K. HEILBRUN, N.E.S. GOLDSTEIN et R.E. REDDING (dir.), Juvenile Delinquency: Prevention, Assessment, and Intervention, New York, Oxford University Press, vol. XIV, p. 19-44.

DEMBO, R., L. WILLIAMS, E.D. WISH, E. BERRY, A. GETREU, M. WASHBURN et al. (1990). «Examination of the relationships among drug use, emotional/psychological problems, and crime among youths entering a juvenile detention center ", The International Journal of Addictions, vol. 25, $\mathrm{n}^{\circ} 11$, p. 1301-1340.

DIAGNOSTICAND STATISTICAL MANUAL OF MENTAL DISORDERS (1994). 4e éd., Washington (DC), American Psychiatric Publishing, p. 886.

DOMALANTA, D.D., W.L. RISSER, R.E. ROBERTS et J.M.H. RISSER (2003). «Prevalence of depression and other psychiatric disorders among incarcerated youths ", Journal of the American Academy of Child and Adolescent Psychiatry, vol. 42, $\mathrm{n}^{\mathrm{O}}$ 4, p. 477-484.

DUBE, S.R., R.F. ANDA, V.J. FELITTI, D.P. CHAPMAN, D.F. WILLIAMSON et W.H. GILES (2001). "Childhood abuse, household dysfunction, and the risk of attempted suicide throughout the life span: Findings from the adverse childhood experiences study ", Journal of the American Medical Association, vol. 286, $\mathrm{n}^{\circ} 24$, p. 3089-3096.

ESPOSITO, C.L. et G.A. CLUM (2002). "Psychiatric symptoms and their relationship to suicidal ideation in a high-risk adolescent community sample», Journal of the American Academy of Child et Adolescent Psychiatry, vol. 41, no 1, p. 44-51.

EVANS, W.P., R.M. MARTE, S. BETTS et B. SILLIMAN (2001). «Adolescent suicide risk and peer-related violent behaviors and victimization", Journal of Interpersonal Violence, vol. 16, $\mathrm{n}^{\circ} 12$, p. 1330-1348.

FARAND, L., F. CHAGNON, J. RENAUD, et M. RIVARD (2004). "Completed suicides among Quebec adolescents involved with juvenile justice and child welfare services ", Suicide and Life-Threatening Behavior, vol. $34, \mathrm{n}^{\circ} 1$, p. 24-35.
FARRINGTON, D.P. (1994). The Twelfth Jack Tizard Memorial Lecture. The Development of Offending and Antisocial Behaviour from Childhood: Key Findings from the Cambridge Study in Delinquent Development, texte présenté lors de la $11^{\mathrm{e}}$ Conférence européenne de la ACPP.

FERGUSSON, D.M., A.L. BEAUTRAIS et L.J. HORWOOD (2003). «Vulnerability and resiliency to suicidal behaviours in young people», Psychological Medicine, vol. 33, $\mathrm{n}^{\circ} 1$, p. 61-73.

FERGUSSON, D.M., L.J. HORWOOD, E.M. RIDDER et A.L. BEAUTRAIS (2005). "Suicidal behaviour in adolescence and subsequent mental health outcomes in young adulthood", Psychological Medicine, vol. 35, nº 7, p. 983-993.

FLOURI, E. (2005). "Psychological and sociological aspects of parenting and their relation to suicidal behavior », Archives of Suicide Research, vol. 9, n 4, p. 373-383.

FLOURI, E. et A. BUCHANAN (2002). "The protective role of parental involvement in adolescent suicide », The Journal of Crisis Intervention and Suicide Prevention, vol. $23, \mathrm{n}^{\mathrm{o}} 1$, p. 17-22.

FOMBONNE, E. (1998). "Suicidal behaviors in vulnerable adolescents: Time trends and their correlates », British Journal of Psychiatry, $\mathrm{n}^{\circ} 173$, p. 154-159.

FOMBONNE, E., G. WOSTEAR, V.COOPER, R. HARRINGTON et M. RUTTER (2001). "The Maudsley long-term follow-up of child and adolescent depression: 2. Suicidality, criminality and social dysfunction in adulthood ", British Journal of Psychiatry, vol. $179, \mathrm{n}^{\circ} 3$, p. $218-223$.

GALLAGHER, C.A. et A. DOBRIN (2006). "Deaths in juvenile justice residential facilities », Journal of Adolescent Health, vol. 38, $\mathrm{n}^{\circ} 6$, p. 662-668.

GOLDSTEIN, N.E.S., O. OLUBADEWO, R.E. REDDING et F.J. LEXCEN (2005). «Mental health disorders: The neglected risk factor in juvenile delinquency ", dans K. HEILBRUN (dir.), Juvenile Delinquency: Prevention, Assessment, and Intervention, New York, Oxford University Press, vol. XIV, p. 85-110.

GOLDSTEIN, T.R., B. BIRMAHER, D. AXELSON, N.D. RYAN, M.A. STROBER, M.K. GILL et al. (2005). "History of suicide attempts in pediatric bipolar disorder: Factors associated with increased risk ", Bipolar Disorders, vol. 7, n 6, p. 525-535.

HARRIS, T.E. et C.J. LENNINGS (1993). "Suicide and adolescence», International Journal of Offender Therapy and Comparative Criminology, vol. 37, no 3, p. 263-270.

HART, J.L., S.K. O'TOOLE, J.L. PRICESHARPS et T.W. SHAFFER (2007). "The risk and protective factors of violent juvenile offending: An examination of gender differences ", Youth Violence and Juvenile Justice, vol. 5, nº 4, p. 367-384.

HAYES, L.M. (2005). "Juvenile suicide in confinement in the United States: Results from a national survey ", The Journal of Crisis Intervention and Suicide Prevention, vol. $26, \mathrm{n}^{\circ} 3$, p. 146-148. 
HILLS, A.L., B.J. COX, L.A. MCWILLIAMS et J. SAREEN (2005). " Suicide attempts and externalizing psychopathology in a nationally representative sample», Comprehensive Psychiatry, vol. 46, $\mathrm{n}^{\circ}$ 5, p. 334-339.

HOGE, R.D., D.A. ANDREWS et A.W. LESCHIED (1992). "Formulaire ministériel d'évaluation des risques et des besoins au moment de la prise en charge: guide et barème de notation par énoncé », texte présenté au Colloque du tribunal de la famille de Londres.

HOLMES, S.E., J.R. SLAUGHTER et J. KASHANI (2001). "Risk factors in childhood that lead to the development of conduct disorder and antisocial personality disorder », Child Psychiatry et Human Development, vol. 31, nº 3, p. 183-193.

HORESH, N., T. ROLNICK, I. IANCUT, P. DANNON, E. LEPKIFKER, A. APTER et al. (1997). "Anger, impulsivity and suicide risk», Psychotherapy and Psychosomatics, vol. $66, \mathrm{n}^{\circ} 2$, p. 92-96.

HOWARD, J., C.J. LENNINGS et J. COPELAND (2003). "Suicidal behavior in a young offender population ", The Journal of Crisis Intervention and Suicide Prevention, vol. 24, n 3, p. 98-104.

HUKKANEN, R., A. SOURANDER et L. BERGROTH (2003). "Suicidal ideation and behavior in children's homes ", Nordic Journal of Psychiatry, vol. 57, $\mathrm{n}^{\circ}$ 2, p. 131137.

JONSON-REID, M. (2002). «Exploring the relationship between child welfare intervention and juvenile corrections involvement ", American Journal of Orthopsychiatry, vol. 72 , no 4, p. 559-576.

KEMPTON, T. et R.L. FOREHAND (1992). "Suicide attempts among juvenile delinquents: The contribution of mental health factors ", Behaviour Research and Therapy, vol. 30, n 5, p. 537-541.

KIDD, S., C.C. HENRICH, K.A. BROOKMEYER, L. DAVIDSON, R.A. KING et G. SHAHAR (2006). "The social context of adolescent suicide attempts: Interactive effects of parent, peer, and school social relations ", Suicide and Life-Threatening Behavior, vol. 36, no 4, p. 386-395.

KING, R.A., M. SCHWAB-STONE, A.J. FLISHER, S. GREENWALD, R.A. KRAMER, S.H. GOODMAN et al. (2001). "Psychosocial and risk behavior correlates of youth suicide attempts and suicidal ideation ", Journal of the American Academy of Child et Adolescent Psychiatry, vol. 40, $\mathrm{n}^{\mathrm{0}}$ 7, p. 837-846.

KOTILA, L. et J. LÖNNQVIST (1988). "Adolescent suicide attempts: Sex differences predicting suicide", Acta Psychiatrica Scandinavica, vol. 77, n 3 , p. 264-270.

KRISBERG, B. et A.M. WOLF (2005). «Juvenile offending ", dans K. HEILBRUN (dir.), Juvenile Delinquency: Prevention, Assessment, and Intervention, New York, Oxford University Press, vol. XIV, p. 67-84.

LAHEY, B.B., I.D. WALDMAN et K. MCBURNETT (1999). «The development of antisocial behavior: An integrative causal model», Journal of Child Psychology and Psychiatry, vol. 40, n 5, p. 669-682.

LANGHINRICHSEN-ROHLING, J., C. ARATA, D. BOWERS, N. O'BRIEN et A. MORGAN (2004). "Suicidal behavior, negative affect, gender, and self-reported delinquency in college students», Suicide and Life-Threatening Behavior, vol. 34, $\mathrm{n}^{\circ} 3$, p. 255-266.

LAY, B., W. IHLE, G. ESSER et M.H. SCHMIDT, (2005). "Juvenile-episodic, continued or adult-onset delinquency? ", European Journal of Criminology, vol. 2, $\mathrm{n}^{\mathrm{0}} 1$, p. 39-66.

MARCOTTE, G.V., D. MARCOTTE et T.R.S. BOUFFARD (2002). «The influence of familial support and dysfunctional attitudes on depression and delinquency in an adolescent population ", European Journal of Psychology of Education, vol. 17, $\mathrm{n}^{\circ} 4$, p. 363-376.

MARTTUNEN, M. et M. PELKONEN (2000). "Psychiatric risk factors for adolescent suicide. A review », Psychiatria Fennica, $\mathrm{n}^{\circ} 31$, p. 110-125.

MCGARVEY, E.L., L.A. KRYZHANOVSKAYA, C. KOOPMAN, D. WAITE et R.J. CANTERBURY (1999). «Incarcerated adolescents' distress and suicidality in relation to parental bonding styles », The Journal of Crisis Intervention and Suicide Prevention, vol. 20, n ${ }^{\circ} 4$, p. 164-170.

MCREYNOLDS, L.S., G.A. WASSERMAN, R.E. DECOMO, R. JOHN, J.M. KEATING et S. NOLEN (2008). "Psychiatric disorder in a juvenile assessment center ", Crime and Delinquency, vol. 54, n 2, p. 313-334.

MEMORY, J.M. (1989). «Juvenile suicides in secure detention facilities: Correction of published rates ", Death Studies, vol. 13, $\mathrm{n}^{\circ} 5$, p. $455-463$.

MORRIS, R.E., E.A. HARRISON, G.W. KNOX, E. TROMANHAUSER et al. (1995). "Health risk behavioral survey from 39 juvenile correctional facilities in the United States », Journal of Adolescent Health, vol. $17, \mathrm{n}^{\circ} 6$, p. 334-344.

NOLEN, S., L.S. MCREYNOLDS, R.E. DECOMO, R. JOHN, J.M. KEATING et G.A. WASSERMAN (2008). "Lifetime suicide attempts in juvenile assessment center youth", Archives of Suicide Research, vol. $12, \mathrm{n}^{\circ} 2$, p. $111-123$.

OTZENBERGER, J.H. (2003). «Personality characteristics relating to suicide risk of incarcerated adolescent males as measured by the Millon Adolescent Clinical Inventory and the Suicide Probability Scale », Dissertation Abstracts International. Section B: The Sciences and Engineering, vol. 64, $\mathrm{n}^{\circ}$ 6-B, p. 2934.

OVERHOLSER, J.C., D.M. ADAMS, K.L. LEHNERT et D.C. BRINKMAN (1995). "Self-esteem deficits and suicidal tendencies among adolescents ", Journal of the American Academy of Child et Adolescent Psychiatry, vol. 34, n 7, p. 919-928.

PATTERSON, G.R., B.D. DEBARYSHE et E. RAMSEY (1989). «A developmental perspective on antisocial behavior ", American
Psychologist. Special Issue: Children and Their Development: Knowledge Base, Research Agenda, and Social Policy Application, vol. 44, no 2, p. 329-335.

PENA, J.B. et E.D. CAINE (2006). «Screening as an approach for adolescent suicide prevention », Suicide and Life-Threatening Behavior, vol. 36, n 6, p. 614-637.

PENN, J.V., C.L. ESPOSITO, L.E. SCHAEFFER, G.K. FRITZ et A. SPIRITO (2003). "Suicide attempts and self-mutilative behavior in a juvenile correctional facility », Journal of the American Academy of Child et Adolescent Psychiatry, vol. $42, \mathrm{n}^{\circ}$ 7, p. 762769.

PERKINS, D.F. et G. HARTLESS (2002). "An ecological risk-factor examination of suicide ideation and behavior of adolescents ", Journal of Adolescent Research, vol. $17, \mathrm{n}^{\circ} 1$, p. 3-26.

PLUTCHIK, R. (1995). "Outward and inward directed aggressiveness: The interaction between violence and suicidality", Pharmacopsychiatry, vol. 28, suppl. 2, p. $47-57$.

PLUTCHIK, R. (2000). "Aggression, violence, and suicide", dans R. MARRIS, W.A. BERMAN, L. et M. SILVERMAN (dir.), Comprehensive Textbook of Suicidology, New York, Guilford Press.

PLUTCHIK, R., A.J. BOTSIS et H.M. VAN PRAAG (1995). "Psychopathology, selfesteem, sexual and ego functions as correlates of suicide and violence risk », Archives of Suicide Research, vol. 1, n ${ }^{\circ}$ 1, p. 27-38.

PLUTCHIK, R. et H.M. VAN PRAAG (1990). "Psychosocial correlates of suicide and violence risk », dans H.M. VAN PRAAG, R. PLUTCHIK et A. APTER (dir.), Violence and Suicidality: Perspectives in Clinical and Psychobiological Research, New York, Brumer/Magel, p. 37-65.

PLUTCHIK, R., H.M. VAN PRAAG et H.R. CONTE (1989). "Correlates of suicide and violence risk: III. A two-stage model of countervailing forces ", Psychiatry Research, vol. 28, nº 2, p. 215-225.

PORTZKY, G., K. AUDENAERT, et K. VAN HEERINGEN (2005). "Suicide among adolescents: A psychological autopsy study of psychiatric, psychosocial and personalityrelated risk factors ", Social Psychiatry and Psychiatric Epidemiology, vol. 40, $\mathrm{n}^{\circ} 11$, p. 922-930.

PRINSTEIN, M.J., J. BOERGERS, A. SPIRITO, T.D. LITTLE et W.L. GRAPENTINE (2000). «Peer functioning, family dysfunction, and psychological symptoms in a risk factor model for adolescent inpatients' suicidal ideation severity ", Journal of Clinical Child Psychology, vol. 29, n 3, p. 392-405.

PRONOVOST, J. et D. LECLERC (2002). "L'évaluation et le dépistage des adolescent(e)s suicidaires en centres jeunesse. / Evaluation and tracking of suicidal teenagers in Centres jeunesse", Revue de psychoéducation et d'orientation, vol. 31, p. 1 , p. $81-100$. 
PRONOVOST,J., D. LECLERC, et M. DUMONT (2003). "Facteurs de protection reliés au risque suicidaire chez des adolescents : comparaison de jeunes du milieu scolaire et de jeunes en Centres jeunesse. / Protection factors linked to suicidal risk in adolescents: Comparison of school system teenagers and other teens residing in Youth Centers ", Revue québécoise de psychologie, vol. 24, $\mathrm{n}^{\circ}$ 1, p. 179-199.

PSYCHIATRY, A.A.O.C.A.A. (2001). «Practice parameter for the assessment and treatment of children and adolescent with suicidal behavior", Journal of the American Academy of Child and Adolescent Psychiatry, vol. 40, suppl. 7, p. 24S-51S.

PUTNINS, A.L. (2005). "Correlates and predictors of self-reported suicide attempts among incarcerated youths ", International Journal of Offender Therapy and Comparative Criminology, vol. 49, $\mathrm{n}^{\circ}$ 2, p. 143-157.

RENAUD, J., M.T. BERLIM, A. MCGIRR, M. TOUSIGNANT et G. TURECKI (2008). "Current psychiatric morbidity, aggression/ impulsivity, and personality dimensions in child and adolescent suicide: A case-control study », Journal of Affective Disorders, vol. $105, \mathrm{n}^{\circ} 1-3$, p. 221-228.

RENAUD, J., D.A. BRENT, B. BIRMAHER, L. CHIAPPETTA et J. BRIDGE (1999). "Suicide in adolescents with disruptive disorders", Journal of the American Academy of Child and Adolescent Psychiatry, vol. $38, \mathrm{n}^{\circ} 7$, p. 846-851.

RENAUD, J., F. CHAGNON, G. TURECKI et C. MARQUETTE (2005). "Completed suicides in a youth centres population ", The Canadian Journal of Psychiatry / La Revue canadienne de psychiatrie, vol. $50, \mathrm{n}^{\circ} 11$, p. 690-694.

ROBERTS, A.R. et K. BENDER (2006). "Juvenile offender suicide: Prevalence, risk factors, assessment, and crisis intervention protocols", International Journal of Emergency Mental Health, vol. 8, n ${ }^{\circ} 4$, p. 255-266.
ROHDE, P., D.E. MACE et J.R. SEELEY (1997). "The association of psychiatric disorders with suicide attempts in a juvenile delinquent sample", Criminal Behaviour and Mental Health, vol. 7, n 3, p. 187-200.

ROHDE, P., J.R. SEELEY et D.E. MACE (1997). "Correlates of suicidal behavior in a juvenile detention population », Suicide and Life-Threatening Behavior, vol. 27, $\mathrm{n}^{\circ} 2$, p. 164-175.

RUCHKIN, V.V., M. SCHWAB-STONE, R.A. KOPOSOV, R. VERMEIREN et R.A. KING (2003). "Suicidal ideations and attempts in juvenile delinquents ", Journal of Child Psychology and Psychiatry, vol. 44, $\mathrm{n}^{\circ}$ 7, p. 1058-1066.

RYAN, J.P. et M.F. TESTA (2005). «Child maltreatment and juvenile delinquency: Investigating the role of placement and placement instability", Children and Youth Services Review, vol. 27, n 3, p. 227-249.

SANISLOW, C.A., C.M. GRILO, D.C. FEHON, S.R. AXELROD et T.H. MCGLASHAN (2003). "Correlates of suicide risk in juvenile detainees and adolescent inpatients», Journal of the American Academy of Child and Adolescent Psychiatry, vol. 42, $\mathrm{n}^{\circ} 2$, p. 234-240.

SOURANDER, A., L. HELSTELÄ, A. HAAVISTO et L. BERGROTH (2001). «Suicidal thoughts and attempts among adolescents: A longitudinal 8-year follow-up study ", Journal of Affective Disorders, vol. 63, $\mathrm{n}^{\circ}$ 1-3, p. 59-66.

SPECKENS, A.E.M. et K. HAWTON (2005). "Social problem solving in adolescents with suicidal behavior: A systematic review», Suicide and Life-Threatening Behavior, vol. 35, n 4 , p. 365-387.

STATISTIQUE CANADA (2007). «Services communautaires et placement sous garde des jeunes ", Le Quotidien.

ST-LAURENT, D. et C. BOUCHARD (2004). L'épidémiologie du suicide au Québec: que savons-nous de la situation récente?, Institut national de santé publique du Québec.
ST-LAURENT, D. et M. GAGNÉ, (2008). Surveillance de la mortalité par suicide au Québec: Ampleur et évolution du problème de 1981 à 2006, Institut national de santé publique du Québec.

THOMPSON, M.P., C.-H. HO et J.B. KINGREE (2007). «Prospective associations between delinquency and suicidal behaviors in a nationally representative sample », Journal of Adolescent Health, vol. 40, n ${ }^{\circ}$ 3, p. 232-237.

VERMEIREN, R., M. SCHWAB-STONE, V.V. RUCHKIN, R.A. KING, C. VAN HEERINGEN et D. DEBOUTTE (2003). "Suicidal behavior and violence in male adolescents: A school-based study », Journal of the American Academy of Child et Adolescent Psychiatry, vol. 42, $\mathrm{n}^{\circ}$ 1, p. 41-48.

WASSERMAN, G.A. et L.S. MCREYNOLDS (2006). "Suicide risk at juvenile justice intake», Suicide and Life-Threatening Behavior, vol. 36, $\mathrm{n}^{\circ} 2$, p. 239-249.

WILSON, K.G., J. STELZER, J.N. BERGMAN, M.J. KRAL et al. (1995). "Problem solving, stress, and coping in adolescent suicide attempts », Suicide and Life-Threatening Behavior, vol. 25, n ${ }^{\circ}$, p. 241-252.

WOOLF, A. et S.G. FINK (1985). «Epidemiology of trauma in a population of incarcerated youth ", Pediatrics, vol. 75, $\mathrm{n}^{\circ} 3$, p. 463-468.

ZINGRAFF, M.T., J. LEITER, K.A. MYERS et M.C. JOHNSEN (1993). "Child maltreatment and youthful problem behavior », Criminology, vol. 31, $\mathrm{n}^{\circ} 2$, p. 173-202.

ZLOTNICK, C., B.A. WOLFSDORF, B. JOHNSON et A. SPIRITO (2003). "Impaired self-regulation and suicidal behavior among adolescent and young adult psychiatric inpatients », Archives of Suicide Research, vol. 7, n 2, p. 149-157. 